\title{
Réponses physiologiques par poste de jeu des footballeurs d'élite lors d'un match éprouvant dans un environnement chaud et humide
}

\author{
J.M. BAZABA KAYILOU ${ }^{1,2^{*}}$, Alain BOUSSANA ${ }^{2}$, Judith F. AHOUNOU AIKPE ${ }^{1}$, \\ Pierre H. DANSOU ${ }^{1}$ et Joachim D. GBENOU ${ }^{3}$
}

'Laboratoire de Physiologie de l'Effort (LAPEF), Université d'Abomey Calavi. 01BP 918 Cotonou, Bénin.

${ }^{2}$ Laboratoire de Physiologie de l'Effort et Biomécanique (LAPEFB), Université Marien Ngouabi. BP 69

Brazzaville, R. Congo.

${ }^{3}$ Laboratoire de Pharmacognosie et des outils essentiels (LAPHE), Faculté des Sciences de la Santé, Faculté des Sciences et Techniques, Université d'Abomey Calavi. 01BP 918 Cotonou, Bénin.

*Auteur correspondant ; E-mail : jeanmichelbazaba@gmail.com; Tel : 00242069553635

\section{RESUME}

Malgré la hausse de la température atmosphérique pendant la grande saison de pluies, la Fédération Congolaise de Football (FECOFOOT) organise les matchs à 14 heures. Dans le but d'évaluer les paramètres physiologiques des footballeurs par poste de jeu et la distance totale qu'ils parcourent au cours d'un match éprouvant de football en république du Congo. Un match test a été organisé à une température de $39{ }^{\circ} \mathrm{C}$ et une humidité relative de $60 \%$. La fréquence cardiaque d'effort $(170,4 \pm 8,73 \mathrm{bpm})$, la température corporelle centrale $\left(37,06 \pm 0,20^{\circ} \mathrm{C}\right)$ ainsi que la quantité d'eau consommée au cours du match $(2,28 \pm 0,37 \mathrm{~L})$ des attaquants étaient plus élevées que les autres joueurs. Par contre, la perte hydrique $(3,55 \pm 0,55 \%)$, la dépense énergétique $(868,00$ $\pm 99,52 \mathrm{kcal})$ et la distance totale parcourue $(8,95 \pm 0,47 \mathrm{~km})$ des milieux de terrain étaient supérieures aux autres joueurs. Le football pratiqué dans un environnement chaud et humide entraine une contrainte physiologique élevée responsable de la contre performance des footballeurs.

(C) 2020 International Formulae Group. All rights reserved.

Mots clés : Perte hydrique, performance, footballeur, climat chaud et humide, R. Congo.

\section{Physiological responses by playing position of elite footballers during a challenging match in a hot and humid environment}

\begin{abstract}
Despite the rise in atmospheric temperature during the great rainy season, the Congolese Football Federation (FECOFOOT) organizes the matches at $2 \mathrm{pm}$. For the purpose of evaluating the physiological parameters and the total distance travelled by footballers per playing position during a challenging football match in the republic of Congo. A test match was held in a hot and humid environment with a temperature of $38{ }^{\circ} \mathrm{C}$ and a relative humidity of $60 \%$. The exertion heart rate $(170.4 \pm 8.73 \mathrm{bpm})$, core body temperature $\left(37.06 \pm 0.20^{\circ} \mathrm{C}\right)$ and the amount of water consumed during the match $(2.28 \pm 0.37 \mathrm{~L})$ of the strikers were higher than those of the other players. In contrast, water loss $(3.55 \pm 0.55 \%)$, energy expenditure $(868.00 \pm 99.52 \mathrm{kcal})$ and total distance travelled $(8.95 \pm 0.47 \mathrm{~km})$ from midfielders were higher than other players. Football played in a hot and humid environment leads to a high physiological stress responsible for the counter-performance of footballers.
\end{abstract} (C) 2020 International Formulae Group. All rights reserved.

Keywords: Water loss, performance, football player, hot and humid climate, R. Congo. 


\section{INTRODUCTION}

Le football est l'un des sports les plus populaires au monde. Il est pratiqué à différentes catégories d'âge. C'est un sport balistique opposant deux équipes de 11 joueurs et le match se joue pendant au moins 90 minutes dans les compétitions officielles (Yang et al., 2013). Le jeu nécessite un niveau élevé des capacités techniques, tactiques et physiques. Les participants présentent des caractéristiques physiques et physiologiques particulières. Les joueurs de football sont repartis en quatre postes de jeu au cours du match à savoir l'attaque, le milieu de terrain, la défense et le gardien de but. La distance totale parcourue, l'intensité et le temps d'activité au match de football diffèrent en fonction du poste de jeu. De nombreuses études ont examiné les différentes caractéristiques physiques et physiologiques des footballeurs en fonction de poste de jeu (Yang et al., 2013).

En effet, outre les capacités physiques et le niveau d'entraînement, l'environnement a un effet déterminant sur la performance du footballeur. La capacité à réaliser des exercices dans un environnement chaud est réduite par rapport à un environnement neutre (Maughan et Shirreffs, 2008). Le mécanisme responsable de cette réduction comprend une variété de changements dans l'organisme qui se produisent parallèlement à l'augmentation de la température centrale du corps. Une perte de masse corporelle de 1 à $2 \%$ peut exacerber la souche thermique et cardiovasculaire, ce qui nuit à la performance sportive (Hoffman et al., 2005 ; Sawka et al., 2007). Certaines études suggèrent que les besoins en liquide et les pertes d'eau par sudation varient d'un footballeur à un autre (Godek et al., 2008). Il a été observé que, en fonction de la taille et de la masse corporelle, les joueurs de ligne perdent de grandes quantités de sueur, ce qui nécessite l'apport d'une grande quantité d'eau pour compenser les pertes d'eau par sudation (Godek et al., 2008).

En république du Congo, lors de la grande saison de pluies, les matches du championnat national d'élite sénior se jouent la journée à une température proche de $40{ }^{\circ} \mathrm{C}$ et une humidité dépassant $60 \%$. A cela s'ajoute l'insuffisance de terrain d'entraînement. La plupart du temps, les équipes utilisent les mêmes espaces sous une chaleur extrême au cours de la journée. En plus, les footballeurs arrivent à l'entraînement avec une faible quantité d'eau, alors qu'il n'existe pas de point d'approvisionnement en eau pour se réhydrater. Ce qui affecterait l'organisme du footballeur et induirait une contre-performance sportive au cours de l'entraînement.

Au regard des exigences physiques au football et des conditions environnementales, la détermination du statut hydrique des footballeurs exerçant dans un environnement chaud et humide s'avère indispensable. Par conséquent, le but de cette étude était d'évaluer les paramètres physiologiques des footballeurs et la distance totale qu'ils parcourent au cours $\mathrm{du}$ match éprouvant selon le poste de jeu.

\section{MATERIEL ET METHODES}

Une équipe de football a été retenue par la méthode non probabiliste parmi les cinq premières du championnat national de football d'élite congolaise. Pour participer à l'étude, les footballeurs devraient satisfaire aux critères suivants : avoir une licence sportive valide, avoir une durée de 2 ans de pratique du football d'élite, résider en République du Congo pendant les deux dernières années, avoir l'âge compris entre 18 et 30 ans, donner son consentement libre. Les footballeurs présentant des traumatismes ont été exclus de l'échantillon. Ainsi, un effectif de 18

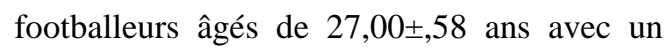
indice de masse corporelle de 22,46 $\pm 1,25$ $\mathrm{kg} / \mathrm{m}^{2}$ a été retenu pour participer à l'étude.

\section{Approche expérimentale}

Un match pré-expérimental a permis aux sujets de se familiariser avec les appareils de mesure au cours d'une séance d'entraînement. Ensuite un match 
expérimental de football a été organisé au cours de la saison sportive.

Le match test s'est déroulé comme un match officiel en deux périodes entre deux équipes composées d'une part des joueurs titulaires et d'autre part des joueurs remplaçants et ceux non retenus dans le cadre de cette étude. L'intensité du match était comprise entre 50 et $80 \%$ de la fréquence cardiaque selon le poste de jeu. La durée du match a été fixée à 90 minutes y compris l'échauffement de 15 minutes.

Cependant, en cas de blessure d'un joueur retenu pour l'expérimentation, les joueurs remplaçants non inclus dans l'échantillon ont complété les effectifs pour permettre l'équilibre des forces entre les deux équipes. Le match test s'est déroulé au sud de la république du Congo (Brazzaville), pendant la grande saison de pluies à une température de $39{ }^{\circ} \mathrm{C}$ et une humidité relative de $60 \%$. L'expérimentation a eu lieu lors de la trêve hivernale du championnat national de football d'élite.

Le jour de l'expérimentation, deux heures avant le match, un repas riche en glucide et protéines a été fourni aux joueurs. Ils étaient astreints de ne pas consommer l'alcool, le café le jour précédant l'expérimentation. La fréquence cardiaque, la température corporelle centrale et la masse corporelle ont été obtenues avant et à la fin du match. Le profil d'activité pendant le match a été évalué à l'aide d'un accéléromètre Garmin Forerunner R15 Quick Start (USA). Avant l'échauffement, les joueurs étaient équipés d'un accéléromètre dont la ceinture est portée au tour de la cage thoracique et la montre portée au poignet, mis en marche, capable d'enregistrer en continu sans interférence la distance totale parcourue, la dépense énergétique et la fréquence cardiaque au cours du match. Il était calibré pour chaque sujet. La distance totale parcourue comprenait toutes les catégories de déplacements (marche, course lente, course en reculons, course accélérée, déplacement latéral).
La température environnementale a été obtenue à l'aide d'un hygromètre électronique SUNROAD (Chine). Au début du match, la température ambiante était de $39{ }^{\circ} \mathrm{C}$, une humidité relative de $60 \%$ et une vitesse de vent de $9 \mathrm{~km} \cdot \mathrm{h}^{-1}$. A la fin du match, la température ambiante était de $37{ }^{\circ} \mathrm{C}$, une humidité relative de $50 \%$ et une vitesse de vent de $11 \mathrm{~km} / \mathrm{h}$. Les procédures pré jeu et de la mi-temps, ainsi que le coaching étaient similaires aux scenarios d'un match officiel. Les valeurs de la fréquence cardiaque, de la température centrale corporelle et de la perte hydrique ont été obtenues avant et à la fin du match. Le joueur était libre de boire par rapport à sa soif en faisant signe à l'équipe expérimentale, qui avait la gestion des bouteilles d'eau. Les bouteilles étaient étiquetées par joueur pour le besoin de contrôle et de suivi. La quantité d'eau consommée lors de l'échauffement était prise en compte, à la fin du match les bouteilles étaient regroupées par joueur pour déterminer la quantité d'eau consommée.

Un pré enquête a été menée aux heures d'entraînement pour observer leur habitude d'apport liquidien. L'étude a été approuvée par le comité scientifique de l'université Marien Ngouabi (Congo Brazzaville) est en accord avec le code de déontologie de l'International Journal des Sports et de Médecine. La fréquence cardiaque de repos a été prise pour chaque sujet, en position assise après 15 minutes de repos. Elle a été obtenue à l'aide d'un cardio-fréquencemètre (polar RS 100 running, France). La température buccale a été prise chez chaque joueur en utilisant le thermomètre électronique (TEMP'10, UE), la température relevée est réajustée de $+0,4{ }^{\circ} \mathrm{C}$ pour correspondre à la température rectale. En ce qui concerne la perte hydrique, plusieurs méthodes sont disponibles pour évaluer l'état d'hydratation, chacun ayant des limites en fonction de la façon dont les liquides sont perdus (Cheuvront et al., 2008 ; Cheuvront et Kenefick, 2014). Dans le cadre de cette étude, nous avons déterminé le pourcentage de perte hydrique en pesant les joueurs avant et à la fin du match semi-nus (avec sous vêtement) à 
l'aide d'un impédancemètre à multifonctions (TANITA Corporation BC-545N, JAPAN).

\section{Analyse statistique}

Le test de Wilcoxon a été utilisé pour comparer les paramètres avant et après match. Une analyse de variance (Friedman) à deux facteurs (groupe vs mesures) pour comparer les valeurs prises avant et après entre les groupes. Lors que l'analyse de Friedman était significative, le test de Wilcoxon a été utilisé pour la comparaison deux à deux. Une analyse de corrélation de Pearson a été utilisée pour déterminer les relations entre les variables de l'étude. Le niveau de significativité des tests statistiques a été fixé à $\mathrm{p}<0,05$. Toutes les analyses ont été effectuées à l'aide du logiciel IBM SPSS Statistics v 22 IBM corporation, USA.

\section{RESULTATS}

La fréquence cardiaque a augmenté à l'effort pour tous les footballeurs quelques soit le poste de jeu. L'intensité du match était de $52 \%$ pour les gardiens de but, $83 \%$ pour les défenseurs, $80 \%$ pour les attaquants et les milieux de terrain (Tableau1). La comparaison des résultats inter groupe montre une dominance des valeurs des défenseurs avec une différence significative $(\mathrm{p}<0,05)$ La température corporelle centrale des footballeurs pendant le match a augmenté quel que soit le poste de jeu. Il était de $0,9{ }^{\circ} \mathrm{C}$ pour les défenseurs, $0,8^{\circ} \mathrm{C}$ pour les gardiens de but, $0,92{ }^{\circ} \mathrm{C}$ pour les milieux de terrain et $1,3{ }^{\circ} \mathrm{C}$ pour les attaquants (Tableau 2). La comparaison des valeurs inter groupe a montré des valeurs plus élevées chez les attaquants avec une différence significative $(\mathrm{p}=0,021)$.

En ce qui concerne la perte hydrique, les résultats obtenus montrent un pourcentage de perte hydrique $1,5 \%$ pour les gardiens de but, $3,33 \%$ pour les défenseurs, $3,55 \%$ pour les milieux de terrain et $2,83 \%$ pour les attaquants. La comparaison inter groupe montre un pourcentage de perte hydrique plus élevé chez les milieux de terrain (Tableau 3). Par ailleurs, pour la quantité d'eau consommée au cours du match (Tableau 3), les résultats obtenus montrent que les attaquants ont consommé plus d'eau par rapport aux autres joueurs $(\mathrm{p}=$ 0,034).

La dépense énergétique pendant le match par poste était de 794,80 $\pm 0,11 \mathrm{kcal}$ pour les défenseurs, $649,60 \pm 18,18 \mathrm{kcal}$ pour les gardiens de but, $868,00 \pm 99,52 \mathrm{kcal}$ pour les milieux de terrain et $834,00 \pm 75,04 \mathrm{kcal}$ pour les attaquants (Tableau 3 ). Ces résultats ont montré que les milieux du terrain ont dépensé plus d'énergie au cours du match que les autres joueurs $(\mathrm{p}<0,001)$.

La distance totale parcourue au cours du match était de 7,88 $\pm 0,44 \mathrm{~km}$ pour les défenseurs, 4,52 $\pm 0,60 \mathrm{~km}$ pour les gardiens de buts, $8,95 \pm 0,47 \mathrm{~km}$ pour les milieux du terrain et $8,22 \pm 0,47 \mathrm{~km}$ pour les attaquants (Tableau 3). La comparaison des résultats montre que les milieux de terrain ont parcouru une plus longue distance au cours du match par rapport aux autres joueurs $(p<0,001)$.

Tableau 1: Comparaison des paramètres physiologiques de match des footballeurs par poste de jeu.

\begin{tabular}{llll}
\hline Poste de jeu & FCt $(\mathbf{b p m})$ & FCE $(\mathbf{b p m})$ & \% FC = intensité \\
\hline Gardien1 & 192 & 153 & 52 \\
Gardien2 & 192 & 152 & 51 \\
Défenseurs $(\mathrm{n}=5)$ & $192,12 \pm 1,80$ & $166,4 \pm 9,93$ & $82,6 \pm 1,81^{*}$ \\
Milieux $(\mathrm{n}=5)$ & $189,00 \pm 2,34$ & $160,4 \pm 3,43$ & $79,78 \pm 1,82$ \\
Attaquants $(\mathrm{n}=6)$ & $192,50 \pm 3,30$ & $170,4 \pm 8,73^{*}$ & $79,93 \pm 1,82$ \\
\hline
\end{tabular}

FCE: Fréquence cardiaque maximale, FCt: Fréquence cardiaque théorique. 
Tableau 2 : Comparaison de la température centrale après match par poste de jeu.

\begin{tabular}{llcc}
\hline Poste de jeu & \multicolumn{2}{c}{ Température centrale $\left(\mathbf{T C},{ }^{\circ} \mathbf{c}\right)$} \\
& \multicolumn{1}{c}{ Avant match } & Fin match & $\boldsymbol{\Delta}$ TCE \\
\hline Gardien 1 & 36,09 & 36,89 & 0,8 \\
Gardien 2 & 36,04 & 36,86 & 0,56 \\
Défenseurs $(\mathrm{n}=5)$ & $36,04 \pm 0,50$ & $37,04 \pm 0,09$ & $0,9 \pm 0,41$ \\
Milieux $(\mathrm{n}=5)$ & $36,14 \pm 0,62$ & $37,06 \pm 0,20$ & $0,92 \pm 0,42$ \\
Attaquants $(\mathrm{n}=6)$ & $36,35 \pm 0,20$ & $37,65 \pm 0,16^{*}$ & $1,3 \pm 0,04^{*}$ \\
\hline TC : température centrale ; $\Delta$ TCE : variation de la température centrale à l'effort ; : Différence significative à $\mathrm{p}<0,05$
\end{tabular}

Tableau 3: Pourcentage hydrique, quantité d'eau, dépense énergétique et la distance totale parcourue pendant le match.

\begin{tabular}{lllll}
\hline Poste de jeu & P. Hydrique (\%) & QE $(\mathbf{L})$ & DE $($ Kcal $)$ & DP $(\mathbf{k m})$ \\
\hline Gardien 1 & 1,48 & 1,80 & 649,60 & 4,50 \\
Gardien 2 & 1,50 & 1,70 & 665,18 & 4,70 \\
Défenseur $(\mathrm{n}=5)$ & $3,33 \pm 0,36$ & $1,84 \pm 0,11$ & $794,80 \pm 0,11$ & $7,88 \pm 0,44$ \\
Milieux $(\mathrm{n}=5)$ & $3,55 \pm 0,55^{*}$ & $2,00 \pm 0,29$ & $868,00 \pm 99,52^{*}$ & $8,95 \pm 0,47^{*}$ \\
Attaquant $(\mathrm{n}=6)$ & $2,83 \pm 0,37$ & $2,28 \pm 0,37^{*}$ & $834,00 \pm 75,04$ & $8,22 \pm 0,47$ \\
\hline
\end{tabular}

QE : Quantité d'eau consommée ; DE : Dépense énergétique ; DP Distance totale parcourue ; * : Différence significative

\section{DISCUSSION}

La présente étude a été mise en œuvre pour évaluer les paramètres physiologiques par poste de jeu des footballeurs d'élite congolaise et la distance qu'ils parcourent au cours d'un math éprouvant. Les résultats ont montré des différences des valeurs des paramètres physiologiques et de la distance totale parcourue au cours du match par poste de jeu.

$\mathrm{Au}$ cours de l'exercice, le système cardiovasculaire doit s'adapter à la demande métabolique de l'organisme pour fournir l'oxygène et les substrats énergétiques aux muscles en activité (Manzi et al., 2013). Par ailleurs, il permet de déterminer l'intensité du match en fonction de la fréquence cardiaque maximale. Dans cette étude, l'intensité du match a été obtenue par poste de jeu. Les footballeurs ont atteint une intensité de match comprise entre 50 et $80 \%$ de la fréquence cardiaque. Malgré l'intensité élevée de match, les valeurs de la fréquence cardiaque obtenues dans cette étude sont inférieures à celles de la littérature (Bradley et al., 2009 ; 2010). Bien que nos résultats soient inferieurs à certaines données de la littérature, ils corroborent cependant l'étude de Gonzalez-Alonso et al. (2009). De même, la pratique de l'exercice physique sous la chaleur entraîne une perte hydrique par sudation, lorsque l'exercice physique est pratiqué à une intensité optimale.

La perte hydrique a un effet sur la performance du footballeur, surtout sous un climat chaud et humide. La déperdition hydrique a un effet négatif non seulement sur la performance physique mais aussi sur la thermorégulation (Edwards et Noakes, 2009). L'étude réalisée par Ryan et al. (2017) sur 
l'équilibre liquidienne chez les athlètes de sports d'équipes a montré une perte de masse corporelle moyenne supérieure à $2 \%$. L'étude de Kouassi J-P et al. (2019) sur la perte volontaire du poids au cours d'un camp d'entraînement au Judo, a montré qu'une perte du poids de plus de $3 \%$ expose les athlètes à un risque de santé et à la contre-performance. Une étude actuelle a été réalisée pour connaître l'état d'hydratation des footballeurs en ambiance chaude a montré une perte de poids de 2\% (Lusamaki et al., 2014).

Dans le cadre de la présente étude réalisée dans un environnement chaud et humide, les données ont été obtenues par poste de jeu en pesant les joueurs avant et à la fin du match. Nos résultats montrent un pourcentage de perte hydrique de plus de $3 \%$ par poste de jeu. Ces résultats sont supérieurs à ceux des autres études en environnement chaud (RicoSanz et al., 1996). Cela se justifie par les conditions environnementales. Le match expérimental s'est joué sur un terrain synthétique, la chaleur atmosphérique associée à la chaleur chimique du matériel synthétique exposé à la chaleur environnementale est responsable de l'hypo-hydratation. Ce qui entraîne une contrainte thermique qui pousse les joueurs à refroidir leurs pieds au cours du match en les plongeant dans des sceaux d'eau. En plus, les sujets de l'étude se trouvaient dans un état d'hypo-hydratation au jour le jour par les mauvaises habitudes d'hydratation à l'entraînement. Cet état d'hypo-hydratation a eu des répercussions sur les paramètres physiologiques et la distance totale parcourue. A ce jour, la majorité des études sont potentiellement limitées par leur méthode (Cotter et al., 2014).

La hausse de température corporelle qui accompagne la déshydratation exerce une tension sur les mécanismes thermorégulateurs de l'organisme, ce qui augmente le risque de malaise causé par la chaleur et l'effort (Casa, 1999 ; Casa et al., 2007) et réduit la capacité à performer de façon optimale (Huggins et al., 2012). De plus, un taux élevé de déshydratation entraine une diminution $\mathrm{du}$ volume plasmatique et du taux de sudation, ce qui réduit, par évaporation, la sueur pendant un exercice effectué dans un environnement chaud (Montain et al., 2010).

Dans des conditions «agressives », l'organisme se trouve confronté à un problème : le flux sanguin artériel doit apporter du dioxygène vers les muscles actifs, tandis que le flux sanguin cutané doit lui aussi être augmenté, pour préserver la température centrale en augmentant la sudation. Une compétition se met en place entre ces 2 besoins majeurs, celui de la performance motrice et celui de la régulation thermique centrale. Normalement, pour éviter une hyperthermie fatale, le besoin de régulation thermique prime, ce qui explique l'effondrement de la performance, par abaissement du débit sanguin musculaire (Laurent, 2019).

Dans cette étude réalisée dans un environnement chaud et humide, seule la température centrale a été prise en compte. La plupart des travaux antérieurs ont mesuré la température corporelle, ceux tenant compte de la température centrale sont rares à notre connaissance. Nos résultats sont plus faibles à ceux des autres auteurs (Casa et al., 1999; Huggins et al., 2012). Plusieurs explications justifient cette différence. La nature de l'activité physique, le football est un sport intermittent ou l'athlète a la possibilité individuelle de récupération hormis la récupération collective accordée à la mi-temps de $15 \mathrm{~min}$ au moins selon les lois de jeu de la FIFA (2014). Les conditions de collecte des données, car les joueurs étaient autorisés de boire en cas de soif, en faisant signe à l'équipe de recherche. Des corrélations positives ont été observées entre la perte hydrique, la température corporelle et la distance totale parcourue.

Plusieurs études portant sur l'observation des actions de match en situation de compétition sont disponibles dans la littérature (Mohr et al., 2010 ; Rampinini et al., 2011). Certaines études ont obtenu des valeurs 
de la distance totale parcourue au cours du match comprises entre 8 et $11 \mathrm{~km}$ (Mohr et al., 205, 2010). D'autres par contre ont rapporté des distances totales de match situées entre 8 et 13km (Bangsbo et al., 1991 ; Di Salvo et al., 2007). Cette différence s'explique par les techniques d'observations utilisées. Nos résultats sont similaires à ceux de Cazorla et al. (2014). Ces résultats montrent que les distances les plus longues sont parcourues par les milieux de terrain et les défenseurs arrière latéraux, alors que les arrières centraux parcourent les distances les plus courtes.

\section{Application pratique}

L'évaluation des paramètres physiologiques et physiques de match peut être utilisée pour le suivi des footballeurs afin de préserver leur état de santé et aboutir à une bonne performance. Par ailleurs, la pratique du football dans un environnement chaud, demande une attention particulière de la part des entraîneurs, des scientifiques du sport et des organisateurs des compétitions sportives. Des mesures exceptionnelles devraient être prises avant de programmer une compétition sportive dans un environnement extrême. En plus, susciter auprès de footballeurs des bonnes habitudes d'apport hydrique avant, pendant et après match pour éviter l'hypo-hydratation responsable de la contre-performance et d'un dysfonctionnement physiologique de l'organisme.

\section{Conclusion}

Une perte hydrique très importante par poste de jeu supérieure à $2 \%$ a été observée, malgré la quantité d'eau consommée par les footballeurs au cours du match. Les sujets de l'étude se trouvaient dans un état d'hypohydratation permanent accumulé pendant les entraînements. Ce qui est dû aux mauvaises habitudes d'apport hydrique pendant la pratique de l'exercice physique. Cet état d'hypohydratation influence négativement la performance physique des footballeurs. Les footballeurs ont consommé une quantité moyenne d'eau importante au cours du match de 2,00 $\pm 0,29 \mathrm{~L}$, qui n'a pas contribué à compenser les pertes liquidiennes par sudation.

\section{CONFLIT D'INTERETS}

Les auteurs de ce manuscrit déclarent qu'ils n'ont aucun conflit d'intérêts y relatif.

\section{CONTRIBUTIONS DES AUTEURS}

JMBK : auteur correspondant, responsable du projet. $\mathrm{AB}$ : collecte des données. JFAA: protocole expérimental. PHD : lecture du manuscrit. JDG : traitement statistique des données.

\section{REMERCIEMENTS}

Nos remerciements vont à l'endroit de la Fédération Congolaise de Football (FECOFOOT), à 1'Institut Supérieur d'Education Physique et Sportive (ISEPS) de la république du Congo, à l'Institut National de la Jeunesse d'Education Physique et du Sport (INJEPS) du Bénin.

\section{REFERENCES}

Bangsbo J, Norregaard L, Thorsoe F. 1991. Activity profile of competition soccer. Can J Sport Sci, 16 (2): 110-116.

Bradley PS, Di Mascio M, Peart D, Olsen P, Sheldon B. 2010. High-intensity activity profiles of elite soccer players at different performance levels. J Strength Cond Res, 24(9):

2343-2351.

Doi:10.1519/JSC.0b013e3181aeb1b3.

Bradley PS, Sheldon W, Wooster B, Olsen P, Boanas P, Krustrup P. 2009. Highintensity running in English FA Premier League soccer matches. J Sports Sci, 27(2): $\quad$ 159-168. Doi: 10.1080/02640410802512775

Casa DJ, Becker SM, Ganio MS, Brown CM, Yeargin SW, Roti MW, Siegler J, Blowers JA, Glaviano NR, Huggins RA, Armstrong LE, Maresh CM. 2007. Validité des appareils qui évaluent la température corporelle pendant l'exercice 
extérieur dans la chaleur. J Athl Train, 42 : 333-342.

Casa DJ. 1999. Exercise in the heat. II. Critical concepts in rehydration, exertional heat illnesses, and maximizing athletic performance. J Athl Train, 34: 253-262.

Cazorla G, Zazoui M, Zahi B, Osmane BN. 2014. Etude de la charge physique d'un match de football. Science et football. Sci Foot, 103-120.

Cheuvront SN, Carter R III, Sawka MN. 2003. Fluid balance and endurance exercisePerformance. Curr Sports Med Rep, 2(4): 202-208. Doi: 10.1249/00149619-200308000-00006

Cheuvront SN, Kenefick RW. 2014. Dehydration: physiology, assessment, and performance effects. Compr Physiol, 4(1): 257-285. Doi: 10.1002/cphy.c130017

Cotter JD, Thornton SN, Lee JKW, Laursen PB. 2014. Sommes-nous noyés dans les conseils d'hydratation? Dommage pour plus? Extrem Physiol Med, 3: 18. Doi: 10.1186/2046-7648-3-18

Di Salvo V, Baron R, Tschan H, Calderon Montero F, Bachl N, Pigozzi F. 2007. Performance characteristics according to playing position in elite soccer. Int $J$ Sports Med, 28: 222-227. Doi: 10.1055/s2006-924294

Edwards AM, Noakes TD. 2009. Dehydration: cause of fatigue or sign of pacing in elite soccer? Sports Med., 39(1): 1-13. Doi: 10.2165/00007256-200939010-00001

FIFA. 2014. Lois du Jeu approuvées et adoptées par l'International Football Association. Board - Zurich.

Godek SF, Bartolozzi R, Burkholder E, Sugarman, Peduzzi C. 2008. Sweat rates and fluid turnover in professional football players: A comparison of National Football League linemen and backs. $J$ Ath Train., 43: 184-189. Doi: 10.4085/10626050-43.2.184

Gonzalez RR, Cheuvront SN, Montain SJ, Goodman DA, Blanchard LA, Berglund
LG, Sawka MN. 2009. Expanded prediction equations of human sweat loss and water needs. J Appl Physiol, 107: 379-388.

Doi: 10.1152/japplphysiol.00089.2009.

Hoffman JR, Kang J, Ratamess NA, Faigenbaum AD. 2005. Réponses biochimiques et hormonales au cours d'une saison de football intercollégial. Med Sci Sports Exerc, 37(7): 1237-1241. Doi:

10.1249/01.mss.000170068.97498.26

Huggins R, Martschinske J, Applegate K. Armstrong L, Casa D. 2012. Influence of dehydration on internal body temperature changes during exercise in the heat: A meta-analysis. Med Sci Sports Exerc, 44: 791.

Kouassi J-P, Kouamé N, Tonon BA, Gouthon $\mathrm{P}$, Nouatin BK, Tako A. Effet de la perte rapide volontaire de poids au cours d'un camp d'entrainement de deux semaines sur les paramètres hydro-électrolytiques et les performances des judokas de l'élite en Côte d'Ivoire. Int. J. Biol. Chem. Sci., 13(7) : $\quad 3119-3130 . \quad$ Doi : 10.4314/ijbcs.vl3i7.12

Laurent Grélot. 2019. Mondiaux d'athlétisme de Doha: quand le climat ruine les performances et menace les athlètes. AixMarseille Université.

Lusamaki M, Nyongombe U, Batina A, Kayisu K, Losimba L. 2014. Hydration in soccer player of Kisangani under the completion and training seasons. J Exp Biol Agric Sci, 3(2): 151-8. Doi: 10.18006/2015.3(2).151.156

Manzi V, Bovenzi A, Impellizzeri F, Carminati I, Castagna C. 2013. Individual trainingload and aerobic-fitness variables in premiership soccer players during the precompetitive season. $J$ Strength Cond Res, 27: 631-636. Doi: 10.1519/JSC.0b013e31825dbd81.

Maughan RJ, Shirreffs SM. 2010. Dehydration and Rehydration in competative sport. Scand J Med Sci Sports, 20(3): 40- 
7.

Doi:

$10.1111 / \mathrm{j} .1600$

0838.2010.01207.x.

Mohr M, Krustrup P, Bangsbo J. 2005. Fatigue in soccer: a brief review. J Sports Sci, $\mathbf{2 3}$ (6): 593-599. Doi: 10.1080/02640410400021286

Mohr M, Mujika I, Santisteban J, Randers MB, Bischoff R, Solano R, Hewitt A, Zubillaga A, Krustrup P. 2010. Examination of fatigue development in elite soccer in a hot environment: a multiexperimental approach. Scand J Med Sci Sports, 20(3): 125-132. Doi: 10.1111/j.1600-0838.2010.01217.x.

Montain SJ, Sawka MN, Latzka WA, Valeri CR. 1998. Thermal and cardiovascular strain from hypohydration: influence of exercise intensity. Int J Sports Med, 19: 87-91. Doi: 10.1055/s2007-971887

Rampinini E, Bosio A, Ferraresi I, Petruolo A, Morelli A, Sassi A. 2011. Match-related fatigue in soccer players. Med Sci Sports Exerc, 43 (11): 2161-70. Doi: 10.1249/MSS.0b013e31821e9c5c

Rico-Sanz J, Frontera WR, Rivera MA, Rivera-Brown A, Molé PA,
Meredith CN. 1996. Effects of hyperhydration on total body water, temperature regulation and performance of elite young soccer players in a warm climate. Int J Sports Med, 17(2): 85-91. Doi: 10.1055/s-2007-972813

Ryan P, Nuccio Kelly A, Barnes James M, Carter and Lindsay B. 2017. Fuid balance in team sport atlète and the efeet of hypohydration on cognitive, technical and physical performance. Sport Med, 4(10): 1951-1982. Doi: 10.1007/s40279017-0738-7

Sawka MN, Burke LM, Eichner ER, Maughan RJ, Montain SJ, Stachenfeld NS. 2007. American College of Sports Medicine position stand. Exercise and fluid replacement. Med Sci Sports Exerc, 39(2): $\quad 377-390 . \quad$ Doi: 10.1249/mss.0b013e31802ca597

Yang SM, Lee WD, Kim JH, Kim MY, Kim J. 2013. Differences in body components and electrical characteristics between youth soccer players and non-athletes. Health, 5: 1010-1015. Doi:10.4236/health.2013.56134 